\title{
Virtual Reality Technology Application in motion simulation of Roofbolter
}

\author{
Fan YANG ${ }^{1, a}$, Sicong YUAN ${ }^{2, b}$ and Xiangkui JIANG ${ }^{3, c}$ \\ 1College of Mechanical and Electrical Engineering, Xi'an University of Architecture and Technology, \\ 710055, china \\ 2School of Automation, Xian University of Posts and Telecommunications, Xian 71012, China \\ a287374629@qq.com, bysc_3c@163.com, cjxk2002@126.com
}

Keywords: Roofbolter, Virtual reality technology, Open Scene Graph

\begin{abstract}
Aiming at the virtual movement emulation's design scheme of the jumbolter , the jumbolter's digital model is built by the 3D modeling software PRO/E5.0,the research combined the jumbolter with virtual reality technology. Jumbolter simulation virtual environment was simulated by means of 3 dimentional simulation platform OSG. This study used OSG: : Node as node type to give movements to each part accordingly,then it updated command callback parameter pointer through the OSG: : Node under the class of OSG: : NodeCallback to monitor the jumbolter movement currently. Finally made the operator feel operating vividly in the real environment in the nVisionR9.0 software operated by an external device.
\end{abstract}

\section{Introduction}

In recent years, virtual reality technology has been widely applied in medical, games, military, $3 \mathrm{~d}$ reconstruction etc. Virtual reality technology is a combination of various techniques, including real-time 3d computer graphics technology, wide Angle stereo display technology, the observer eye hand tracking technology, touch, force feedback technology and language input and output technology.

Hydraulic anchor drilling machine is also called anchor drilling machine, used as one importance drilling machinery IN rock tunnels, mine roadway bolt support .With the development of infrastructure construction in our country, the requirement of jumbolt is growth, andthe type of it became more .Combine with anchor drilling machine and virtual reality technology will provide powerful help for site construction by making construction personnel before construction and operation under various working conditions of object, topographical and geological conditions and weather environment adaptation. At present, some researchers made tower crane simulation combined with virtual reality technology, and some achievements have been made ${ }^{[1,2,3]}$.

Considering the bad environment and operators' professional skills which can impact the construction period, this paper puts forward the jumbolter virtual training system, which can not only improve operators' professional skills, but also simulate the environment through the computer technology. And then to reduce the impact of the bad construction environment and operators' professional skills.

\section{2. jumbolter model and post-processing}

\section{1 jumbolter three-dimensional model is established.}

Main purpose of the simulation is to achieve the operations in the field such as movement and anchor work. If all the parent-child relationship are be found, the movement is quite complex and the simulation will be very difficult. In view of this, in the case of not affect jumbolter simulation movement[5], the model of the machine is divided into four parts which are chassis, body, girder and the big arm, hydraulic motor and bolt. Three-dimensional model of the jumbolter are shown in figure 1. 


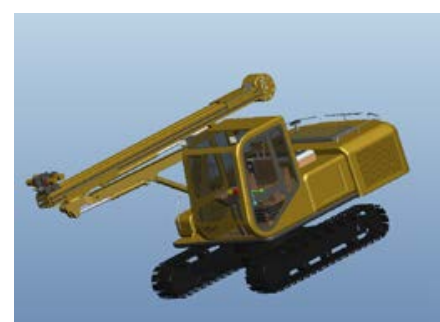

Figure 1 The three-dimensional model of jumbolter

\subsection{The models transformation.}

PRO/E5.0 has very good 3d modeling capabilities, especially suitable for the complex model. But its post processing ability is poor, especially in the appearance of the display and the ability of rendering model is much less than the 3dsMax.3dsMax compared with PRO/E5.0[4], its modeling ability is poor and dimensional precision is low ${ }^{[1]}$. input the model which is build in Pro/E 5.0 into the visual simulation (OSG) platform directly will lead the data loss and incomplete model. This will affect the simulation analysis.

In the view of these reason above, this paper propose a new method that input the model which is build in Pro/E 5.0 into 3ds Mas to output a conversion format *.obj. And then transform this format into *.IVE format which can be accepted by OSG. Finally finish the material setting and compare the missing part which lost in the transform processing to get the complete model. Eventually realize the virtual reality technology can display in nVision R9.0. the whole train of this method is shown in figure 2.

\section{Visual simulation platform OSG system analysis and design}

OSG is an open source efficient 3d graphics development kit, use openGL technology development based on industry standards $\mathrm{c}^{++}$platform application program interface (API), the developer can use it to realize the virtual reality, simulation application, establish virtual $3 \mathrm{~d}$ scene, etc. Its main advantage is that it is free and open source code, as well as its core database contains a variety of practical tools, such as cutting, special effects simulation scene effect for developers to provide the help.

\subsection{The movement of variables.}

Based on the analysis, the operation of anchor drilling machine in virtual simulation system is mainly for getting the jumbolter nodes, movement variables (such as hydraulic lever scale length, Angle of wheel rotation, the car and head on the chute moving distance) of the parameters.

In OSG, the management of the scene graph is top-down and the layered tree-like data structure organize spatial data, in order to improve the efficiency of rendering, OSG mainly includes three types of nodes: Node, the Geode (leaf nodes) and Group (the set of nodes).Through the analysis of virtual simulation of the anchor drilling machine movement a, using the Node type of the Node, osg::Node class inheritance in osg::Object class application, osg::Object class provides a series of used to save and get name, specify the save data is static or dynamic change, so that at the time of the simulation is convenient to find the corresponding Node ${ }^{[6]}$.Set of nodes may have more child nodes, and the program can be orderly arranged its geometry and status data, by the child nodes of the transformation model of different node position, result in different effect.The Group node is shown in figure 2.

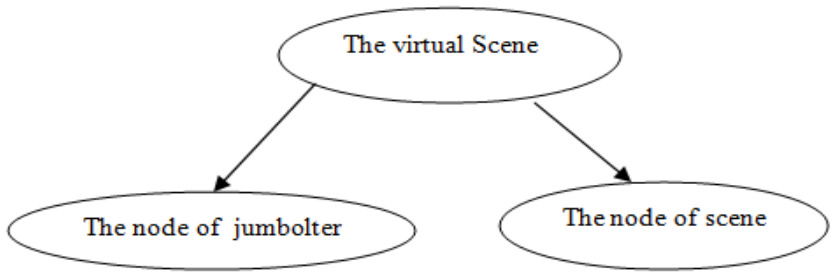

Figure 2 Group node

3.2 The realization of the jumbolter simulation exercise.

After obtaining jumbolter motion parameters, we can simulate the movement of jumbolter in 
virtual reality simulation system. The main components of movement are as follow: the beam of angle, turntable, rotation Angle and swing Angle to the beam and head to the process, the big arm, to beam slide stroke as well as lifting Angle.

In OSG, achieve the transformation of geometric data is mainly depend on OSG transform class, Transform class inheritance Group classes, it can have multiple nodes, but the Transform class is a virtual base class which cannot be instantiated by the application. On this basis, we Can use osg: Materix Transform or osg:: Position Attitude Transform to replace of it[6].

By analysis the movement of jumbolter can get the result that the movement is mainly composed of rotation and translation in the simulation and chose osg:: Materix Transfrom of the above two kinds of nodes. Because this kind of node mainly realizes the child node space transformation matrix, in order to realize the scene object rotation, translation, scaling and so on.osg: : MaterixTransform saved a $4 * 4$ matrix, which contains a total of 16 left by the operation of the commonly used floating point class to achieve the rotation of the node, such as translational movement._MaterixTransform internal use osg::Materix object, to create an execution MaterixTransform node, the first thing to create Materix transformation matrix, and its associated MaterixTransform, then can the implementation model in the simulation system of sports [7-8].

\section{3 jumbolter movement of real-time data update}

Using osg::Node Node under the class of osg:NodeCallback (update the callback parameter pointer) command, and the data of real-time updates can also be understanding into the user's own definition of a function. According to the different methods of correction (update the update, chosen from cull, draw the draw), the callback function run all by themselfs. In all of scene graph traversal, if encounter a node has been associated with a user defined callback class and function,this node will be call back to execute.

In order to achieve real-time dynamic jumbolter, we need to transfer the motion of the nodes function into the corresponding callback function, so that process the chosen and draw .It will use operator () method to get data real-time motion node.

Achieve the jumbolter virtual simulation diagram as shown in figure 3.

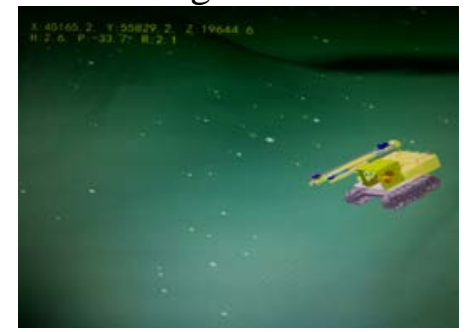

Figure 3 jumbolter in virtual reality

\section{Conclusion}

In this paper, we build the $3 \mathrm{~d}$ model of jumbolter in Pro/E 5.0. and then post process the model in 3ds Max. combine these two point, we can get a model of jumbolter which is required in virtual simulation. And use OSG platform to simulate the model. Finally, combine the OSG and MFC, create the interaction platform of jumbolter. This platform can change the sight angle to meet the users every needs.

\section{Reference}

[1]Yuan sicong,Wang Rong,Li Chao. Virtual reality technology in high-rise tower shaped Monitoring[J]. machine design , 2013,30 (11) : p. 17-21.

[2] Wang Rong. Virtual reality technology tall tower shaped training system based on. Xi'an University of Architecture and Technology.2013

[3]Fu TongQing, Virtual reality technology tower crane 3D simulation training system based on Internet[D]. ShanDong University of Architecture.2012 
[4]Yang ShanGuo.Design of hydraulic bolt rig [D].China University of Mining and Technology.2012

[5]Liu GuiYang,Li SiYing. Sprinkler car virtual simulation design[J]. Agricultural Mechanization Research,2014(12):24-29.

[6]Yang HuaBin OpenSceneGraph 3.0-Three-dimensional visual simulation technology development Comments[M]. National Defense Industry Press.2012

[7]Hou Xiang. Three Dimensional Modeling of virtual reality technology[D]. Chongqing University.2006

[8]Li RongHui. Application of three-dimensional modeling technology in virtual reality techniques[D]. Daqing Petroleum Institute.2007 